\title{
Vegetation Change After 65 Years of Grazing and Grazing Exclusion
}

\author{
Danielle R. Courtois, Barry L. Perryman, and Hussein S. Hussein \\ Authors are rangeland technician, Bureau of Land Management, Socorro, NM 87801; and assistant professor and \\ associate professor, respectively, Department of Animal Biotechnology, University of Nevada, Reno, NV 89557.
}

\begin{abstract}
The Nevada Plots exclosure system was constructed in 1937 following passage of the Taylor Grazing Act to assess long-term effects of livestock grazing on Nevada rangelands. A comparison of vegetation characteristics inside and outside exclosures was conducted during 2001 and 2002 at 16 sites. Data analysis was performed with a paired $t$ test. Out of 238 cover and density comparisons between inside and outside exclosures at each site, $34(14 \%$ of total) were different $(P<0.05)$. Generally, where differences occurred, basal and canopy cover were greater inside exclosures and density was greater outside. Shrubs were taller inside exclosures at 3 sites grazed by sheep (Ovis aries). Perennial grasses showed no vertical height difference. Aboveground plant biomass production was different at only 1 site. Plant community diversity inside and outside exclosures were equal at 11 of 16 sites. Species richness was similar at all sites and never varied $>4$ species at any site. Few changes in species composition, cover, density, and production inside and outside exclosures have occurred in 65 years, indicating that recovery rates since pre-Taylor Grazing Act conditions were similar under moderate grazing and grazing exclusion on these exclosure sites.
\end{abstract}

\section{Resumen}

El sistema de exclusiones de Nevada se construyó en 1937, después de ser aprobada el Acta Taylor de Apacentamiento, para evaluar los efectos a largo plazo del apacentamiento del ganado sobre los pastizales de Nevada. Durante los años 2001 y 2002 , en 16 sitios se condujo una comparación de las características de la vegetación dentro y fuera de las exclusiones. El análisis de los datos se llevo a cabo mediante pruebas de $t$-student para muestras apareadas. De 238 comparaciones de cobertura y densidad de la vegetación dentro y fuera de las exclusiones, 34 de ellas $(14 \%$ del total) fueron diferentes $(P<0.05)$. Generalmente, donde ocurrieron las diferencias, la cobertura basal y de la copa fueron mayores dentro de las exclusiones y la densidad fue mayor fuera de ellas. En tres sitios apacentados con ovinos (Ovis aries) los arbustos fueron mas altos dentro de las exclusiones. Los zacates perennes no mostraron diferencias verticales de altura. La producción de biomasa vegetal aérea fue diferente solo en un sitio. La diversidad de la comunidad vegetal dentro y fuera de las exclusiones fue igual en 11 de los 16 sitios. La riqueza de especies fue similar en todos los sitios y nunca varió por más de 4 especies en ninguno de ellos. Pocos cambios han ocurrido en 65 años en la composición de especies, cobertura, densidad y producción dentro y fuera de las exclusiones, indicando que en estos sitos de exclusión las tasas de recuperación anteriores a las condiciones establecidas por el Acta Taylor fueron similares bajo un apacentamiento moderado y la exclusión al apacentamiento.

Key Words: exclosures, diversity, succession, herbivory effects

\section{Introduction}

Great Basin plant communities have remained essentially unchanged in terms of species composition for at least the last 1.2 million years (Tidwell et al 1972; Barnosky et al 1987). These plant communities have evolved with an assemblage of herbivores including domestic livestock within the last 150 years. There are those among the public that have questioned the use of Great Basin public lands for domestic livestock grazing. Advocates for the removal of livestock often do not provide empirical evidence of deleterious long-term effects from properly managed livestock grazing on western rangelands. Public land livestock grazing supporters likewise have

Research was funded by the Nevada Arid Rangeland Initiative and Nevada Agriculture Experiment Station.

Correspondence: Dr Barry L. Perryman, Dept of Animal Biotechnology, Mail Stop 202, University of Nevada-Reno, Reno, NV 89557. Email: bperryman@cabnr.unr.edu

Manuscript received 9 September 2003; manuscript accepted 5 May 2004. little documented evidence that moderate long-term grazing will not adversely affect Great Basin plant communities.

After the overuse of Great Basin rangelands from the mid1850 s until 1934, rangeland managers sought methods of quantifying the effect of livestock grazing on the landscape. In 1936, the US Forest Service Intermountain Forest and Range Experiment Station, the University of Nevada Agricultural Experiment Station, and the Taylor Grazing Service designed a long-term study to observe secondary plant succession dynamics on overgrazed semiarid ranges in Nevada (Holmgren 1976). Twenty-eight sites were chosen in 9 Nevada counties from 1936 to 1939 on lands then administered by the US Department of Interior, Division of Grazing. These sites became known as the Nevada Plots.

The twenty-eight sites were originally established to study patterns and rates of vegetation change under continued grazing and compared with vegetation within exclosures that were not grazed. Exclosures were located in areas that had been subjected to uncontrolled grazing pressure by domestic animals for 4 to 5 decades prior to 1937 . Each exclosure was about $100 \times 160 \mathrm{~m}$ (1.6 ha) in size (McGinnies 1951). All the exclosures have barbed 
Table 1. Nevada plot exclosure locations, elevations, annual precipitation zone (PZ), GPS coordinates, and dominant plant community descriptions (USDA 2003).

\begin{tabular}{|c|c|c|c|c|}
\hline Location & Elevation (m) & $\mathrm{PZ}(\mathrm{cm})$ & GPS (lat/long) & Plant community description \\
\hline Baker & 1600 & $20-30$ & $38^{\circ} 56^{\prime} 42.32^{\prime \prime} \mathrm{N} 114^{\circ} 03^{\prime} 12.62^{\prime \prime} \mathrm{W}$ & Basin sagebrush/squirreltail \\
\hline Conner's Station & 1846 & $20-30$ & $39^{\circ} 01^{\prime} 55.86^{\prime \prime} \mathrm{N} 114^{\circ} 33^{\prime} 14.06^{\prime \prime} \mathrm{W}$ & Black sagebrush/winterfat \\
\hline Cushman Well & 1208 & $13-18$ & $39^{\circ} 06^{\prime} 26.85^{\prime \prime} \mathrm{N} 119^{\circ} 38^{\prime} 09.35^{\prime \prime} \mathrm{W}$ & Black greasewood/Indian ricegrass \\
\hline Dinner Station & 1789 & $31-39$ & $41^{\circ} 08^{\prime} 35.05^{\prime \prime} \mathrm{N} 115^{\circ} 50^{\prime} 55.84^{\prime \prime} \mathrm{W}$ & Wyoming sagebrush/basin wildrye \\
\hline Lower Squaw Creek & 1411 & $20-30$ & $40^{\circ} 50^{\prime} 55.70^{\prime \prime} \mathrm{N} 119^{\circ} 34^{\prime} 08.72^{\prime \prime} \mathrm{W}$ & Wyoming sagebrush/squirreltail \\
\hline Upper Squaw Creek & 1726 & $20-30$ & $40^{\circ} 53^{\prime} 17.18^{\prime \prime} \mathrm{N} 119^{\circ} 36^{\prime} 46.88^{\prime \prime} \mathrm{W}$ & Low sagebrush/Sandberg bluegrass \\
\hline Newark Valley \#1 & 1909 & $20-30$ & $39^{\circ} 24^{\prime} 48.20^{\prime \prime} \mathrm{N} 115^{\circ} 35^{\prime} 27.64^{\prime \prime} \mathrm{W}$ & Black sagebrush/Indian ricegrass \\
\hline Newark Valley \#2 & 1850 & $20-30$ & $39^{\circ} 25^{\prime} 35.20^{\prime \prime} \mathrm{N} 115^{\circ} 38^{\prime} 18.75^{\prime \prime} \mathrm{W}$ & Winterfat/squirreltail \\
\hline Paradise Valley \#1 & 1339 & $20-30$ & $41^{\circ} 22^{\prime} 18.16^{\prime \prime} \mathrm{N} 117^{\circ} 33^{\prime} 51.57^{\prime \prime} \mathrm{W}$ & Wyoming sagebrush/squirreltail \\
\hline Paradise Valley \#2 & 1472 & $20-30$ & $41^{\circ} 33^{\prime} 43.91^{\prime \prime} \mathrm{N} 117^{\circ} 32^{\prime} 43.03^{\prime \prime} \mathrm{W}$ & Wyoming/low sagebrush/Sandberg bluegrass \\
\hline Pyramid Lake \#1 & 1277 & $13-18$ & $39^{\circ} 50^{\prime} 21.35^{\prime \prime} \mathrm{N} 119^{\circ} 39^{\prime} 23.67^{\prime \prime} \mathrm{W}$ & Basin sagebrush/Indian ricegrass \\
\hline Pyramid Lake \#2 & 1266 & $13-18$ & $39^{\circ} 52^{\prime} 13.50^{\prime \prime} \mathrm{N} 119^{\circ} 38^{\prime} 30.18^{\prime \prime} \mathrm{W}$ & Lahontan sagebrush/squirreltail \\
\hline Zenobia & 1215 & $13-18$ & $40^{\circ} 08^{\prime} 39.37^{\prime \prime} \mathrm{N} 119^{\circ} 44^{\prime} 50.82^{\prime \prime} \mathrm{W}$ & Lahontan sagebrush/squirreltail \\
\hline Wadsworth & 1261 & $13-18$ & $39^{\circ} 39^{\prime} 06.00^{\prime \prime} \mathrm{N} 119^{\circ} 19^{\prime} 21.87^{\prime \prime} \mathrm{W}$ & Bailey greasewood/Indian ricegrass \\
\hline Ward Mountain & 1920 & $31-39$ & $39^{\circ} 08^{\prime} 27.89^{\prime \prime} \mathrm{N} 115^{\circ} 02^{\prime} 47.45^{\prime \prime} \mathrm{W}$ & Black sagebrush/Indian ricegrass \\
\hline Wellington & 1549 & $20-30$ & $38^{\circ} 42^{\prime} 20.70^{\prime \prime} \mathrm{N} 119^{\circ} 18^{\prime} 28.44^{\prime \prime} \mathrm{W}$ & Black sagebrush/squirreltail \\
\hline
\end{tabular}

wire attached to the upper portions of the juniper perimeter posts (3 strands), with a $10-\mathrm{cm}$ square-pattern heavy-gauge wire attached $30 \mathrm{~cm}$ above the soil surface and extending 15-18 $\mathrm{cm}$ below the soil surface (there are a few exceptions to the wire used in the lower portion). Seven of the exclosures also have rodent exclosures on 0.4 ha within the larger exclosure.

The initial sampling of all exclosures in 1937 included an estimation of the proportion of ground cover by species, as well as a density count of the most important perennials (McGinnies 1951; Holmgren 1976). Holmgren noted in his summary report that the original 28 study sites were chosen by the degree of grazing (heavy) that the land had undergone and that changes in the exclosures themselves should be "considered recovery trends from prior probable retrogression trends and the resultant plant communities might be goals for grazing management to aim for, with the knowledge that response may not be as rapid nor the end quite the same under grazing as under the treatment without grazing" (p. 54).

Today, 16 of the original 28 Nevada Plots are intact. Some of the sites have been lost to land swap arrangements between the Bureau of Land Management and private landowners. Other sites have been used by members of the public as cattle or horse holding pens and have lost their value as ecological study areas. A few of the sites have lost some of their integrity because repairs were not implemented, and these sites were excluded.

This study constitutes an assessment of vegetation characteristics inside and outside exclosures that have been subjected to 65 years of protection from large herbivores compared with adjacent areas that have been moderately grazed continuously over the same period.

This project was designed to assess the effects of moderate grazing and grazing exclusion on semiarid plant communities since grazing was regulated in 1937 with passage of the Taylor Grazing Act. Total shrub and herbaceous plant cover and density, cover and density of cheatgrass (Bromus tectorum L.), soil biotic crust cover, vertical height of shrubs and perennial grasses, aboveground plant biomass production, and species richness and diversity were measured and compared inside and outside exclosures.

\section{Materials and Methods}

\section{Study Area}

The Great Basin is the largest temperate semidesert in North America (Miller et al 1994; Morris and Stubben 1994). The ecosystem is characterized primarily by sagebrush steppe grasslands and salt-desert shrubland ecological communities that cover about $44.8 \times 10^{6}$ and $17.6 \times 10^{6}$ ha, respectively (Kuchler 1970; Holecheck et al 1989). Three borders define the physiographic Great Basin: the Sierra Nevada and southern Cascades to the west, the Rocky Mountains on the east, and the Columbia Plateau to the north (Morris and Stubben 1994). The Great Basin contains a series of 33 different mountain ranges that are long, generally narrow, and orientated north to south (Tueller 1989). Sixteen ranges have peaks that reach above $3352 \mathrm{~m}$. Valley floors are between 1615 and $2321 \mathrm{~m}$ in the central and eastern portions of Nevada, but in the northeast, they decline to 1158 to $1767 \mathrm{~m}$ (Morris and Stubben 1994). Sixty percent of precipitation falls primarily as snow in winter and averages 5 to $23 \mathrm{~cm}$ in Nevada (Miller et al 1994). The rain shadow effect prevents much of the Pacific moisture from crossing the Sierra Nevada range; however, summer thundershowers can be heavy and cause localized flooding. Temperatures range from $-22^{\circ} \mathrm{C}$ in winter to $38^{\circ} \mathrm{C}$ in the summer (Tueller 1989). Soils are mostly Aridisols or Entisols and vary in salinity. Upper soil profiles are generally sandy loam, and sometimes nearly all sand, with very low organic content (USDA 1993). Lower portions of the soil profile can contain layers in which clay particles have accumulated. Salts accumulate on the soil surface in depressions in which water has collected with finer sediments from upland sites and has evaporated. Wind removes fine soil particles, leaving a desert pavement comprising small rocks and gravel. 
Table 2. Nevada Plot exclosure allotment grazing histories (current state of knowledge) by site.

\begin{tabular}{|c|c|}
\hline Location & Grazing history \\
\hline Baker & $\begin{array}{l}\text { Winter sheep 1952-1955. Fall/winter sheep 1956-1962. Spring/fall/winter sheep 1963-1967. Winter cattle 1970-1977. } 1978-1980 \text { sheep and } \\
\text { cattle spring/fall/winter. 1981-1986 cattle spring/fall/winter; sheep fall/spring. 2001-2002 } 3758 \text { sheep AUMs from } 1 \text { December to } 30 \text { April and } \\
491 \text { cattle AUMs from } 1 \text { November to } 10 \text { June. }\end{array}$ \\
\hline Conner's Station & $\begin{array}{l}\text { Heavy sheep/cattle use until } 1945.50 \% \text { reduction 1947-1959. 1960-1963 no use. 1964-1965 cattle only winter. Spring cattle 1966-1986 with } \\
\text { much lower stocking rate. 1994-2002 spring cattle } 7572 \text { AUMs. }\end{array}$ \\
\hline Cushman Well & Winter cattle $1937-1970$. No use $1970-1980.1981$ to present, cattle winter range. \\
\hline Dinner Station & $\begin{array}{l}\text { Summer cattle before } 1950 \text { with } 30 \% \text { reduction over period, concurrent spring sheep before } 1965 \text {. Spring/fall cattle 1966-1986. Spring through } \\
\text { fall cattle and spring/early summer sheep } 1987 \text { to present. }\end{array}$ \\
\hline Lower Squaw Creek & $\begin{array}{l}\text { Before } 1965 \text {, cattle, sheep, and feral horse. Continuous cattle } 1965-1975.1974 \text { horse die-off closed allotment until 1982. Spring/fall cattle } 1982- \\
\text { 1986. } 1987 \text { to present, early spring cattle with rest rotation between } 2 \text { pastures. }\end{array}$ \\
\hline Upper Squaw Creek & $\begin{array}{l}\text { Historic sheep trail area, extensively grazed by sheep and cattle. } 1995 \text { to present, spring/summer cattle. } 1982 \text { EIS listed AUM use as: cattle } \\
14054 \text {, horses } 4500 \text {, mule deer } 6340 \text {, antelope } 1150 \text {, and bighorn sheep } 1228 \text {. }\end{array}$ \\
\hline Newark Valley \#1 & $\begin{array}{l}\text { Early } 1900 \text { s to } 1968 \text { heavy sheep use fall/winter/spring. Spring sheep 1969-1976. Fall/winter cattle 1977-1986. } 1987 \text { to present, winter/spring } \\
2465 \text { cattle AUMs and } 1552 \text { sheep AUMs. }\end{array}$ \\
\hline Newark Valley \#2 & $\begin{array}{l}\text { Early } 1900 \text { s to } 1968 \text { heavy sheep use fall/winter/spring. Spring sheep 1969-1976. Fall/winter cattle 1977-1986. } 1987 \text { to present, winter/spring } \\
2465 \text { cattle AUMs and } 1552 \text { sheep AUMs. }\end{array}$ \\
\hline Paradise Valley \#1 & Unknown until 1986. 1986 to present winter/spring 624 cattle AUMs. \\
\hline Paradise Valley \#2 & tle since 1919. Current, spri \\
\hline Pyramid Lake \#1 & Cattle and sheep continuous prior to 1960. Continuous cattle 1961-2000. 2 \\
\hline Pyramid Lake \#2 & $\begin{array}{l}\text { Sheep and cattle winter range until 1960. Spring/summer/fall cattle 1961-1986. Rate as high as } 1023 \text { AUMs 1963-1973. AUMs increasing } \\
\text { since } 1984 .\end{array}$ \\
\hline Zenobia & Continuous sheep prior to 1936. Continuous cattle 1937-1965. Fall/winter/spring cattle 1966-2000. 2001 to present winter cattle. \\
\hline Wadsworth & $\begin{array}{l}\text { Before 1950, used as cattle holding area for rail shipping yards at Wadsworth, NV. Fall/winter cattle 1951-1986. Winter cattle } 1987 \text { to } \\
\text { present. }\end{array}$ \\
\hline Ward Mountain & Winter/spring sheep 1940-1978. Spring/summer alternate years sheep/cattle 1979-1986. Spring/summer cattle 1987 to present. \\
\hline Wellington & ntinuous sheep prior to 1936. Area was sheep trail until early 1940s. Fall/winter cattle 1945-1986. Winter cattle 1987 to present. \\
\hline
\end{tabular}

\section{Description of Exclosure Sites}

The 16, 1.6-ha exclosure sites used in this assessment were located within 7 distinct plant communities (Table 1). Grazing history information was assembled from Bureau of Land Management office archives (Holmgren 1976; Burnside 1988; Table 2). Four of the sites were communities dominated by black sagebrush (Artemisia nova A. Nelson) associated with Indian ricegrass (Achnatherum hymenoides [Roem. \& Schult.] Barkworth) and bottlebrush squirreltail (Elymus elymoides [Raf.] Swezey). Four sites were Wyoming big sagebrush (Artemisia tridentata [Nutt.] subsp. wyomingensis Beetle \& A.L. Young) communities codominant with bottlebrush squirreltail, and 3 were codominant with bud sage (Artemisia spinescens D.C. Eaton.), Indian ricegrass and Great Basin wildrye [Leymus cinereus Scribn. \& Merr.] Á. Löve). Two of the sites were dominated by basin big sagebrush (A. tridentata subsp. tridentata) with bottlebrush squirreltail and Indian ricegrass in the understory. Two sites were dominated by Lahontan sagebrush (Artemisia arbuscula subsp. longicaulis Winward \& McArthur) with a bottlebrush squirreltail understory. Two sites were dominated by greasewood, either Bailey greasewood (Sarcobatus baileyi [Cov.] Jeps.) or black greasewood (Sarcobatus vermiculatus [Hook.] Torr.), with Indian ricegrass understories. The Bailey greasewood site also contained a substantial quantity of Nevada dalea (Psorothamnus polydenius [Torr. ex S. Watts.] Rydb.). One site was a low sagebrush (A. arbuscula Nutt.)-Sandberg bluegrass (Poa secunda J. Presl.) community, and 1 site was essentially a winterfat (Krascheninnikovia lanata [Pursh] A. Meeuse \& A. Smit) monoculture.

At each site, a comparison of parameters inside and outside the exclosure was performed with a paired $t$ test. Percent basal and canopy cover values were arcsine transformed before analysis to achieve normal distribution (Zar 1999); however, values are reported as percentages. Differences were determined to be significant at $P<0.05$ for all analyses. Field sampling was conducted during spring and early summer of 2001 and 2002, prior to the livestock grazing season. The effects of wildlife herbivory and feral horse (Equus caballus) were not controlled inside or outside exclosures; however, no visual evidence of their grazing activity was detected.

Basal cover of herbaceous species and canopy cover of shrubs (by species) were determined by the line intercept method (Canfield 1941), which is valid for measuring shrub communities within central Nevada (Hanley 1978). Within each exclosure, a center point was located and permanently marked with a concrete reinforcing rod. Random compass headings were selected and 3,50-m transects were extended from the central point out toward the perimeter of each 1.6-ha exclosure. Outside the exclosure, 3, 50-m transects were randomly located away from the immediate area surrounding the exclosure (Mueller-Dumbois and Ellenberg 1974; Pieper 
Table 3. Mean (SE) total vegetation cover, density, and probability values $(P)$ inside (In) and outside (Out) exclosures in 2001 and 2002.

\begin{tabular}{|c|c|c|c|c|c|c|c|c|c|}
\hline \multirow[b]{2}{*}{ Location } & \multirow[b]{2}{*}{ Exclosure } & \multicolumn{4}{|c|}{2001} & \multicolumn{4}{|c|}{2002} \\
\hline & & Cover (\%) & $P$ & Density (plants $/ \mathrm{m}^{2}$ ) & $P$ & Cover (\%) & $P$ & Density (plants $/ \mathrm{m}^{2}$ ) & $P$ \\
\hline \multirow[t]{2}{*}{ Baker } & $\ln$ & $2(1)$ & 0.152 & $6(1)$ & 0.403 & $3(2)$ & 0.770 & $5(1)$ & 0.044 \\
\hline & Out & $3(1)$ & & $8(4)$ & & $3(1)$ & & $4(0)$ & \\
\hline \multirow[t]{2}{*}{ Conner's Station } & $\ln$ & $17(3)$ & 0.023 & 7 (2) & 0.073 & 7 (2) & 0.044 & $9(9)$ & 0.720 \\
\hline & Out & $4(1)$ & & $13(1)$ & & $4(0)$ & & $11(3)$ & \\
\hline \multirow[t]{2}{*}{ Cushman Well } & In & $3(2)$ & 0.428 & 2 (1) & 0.485 & $4(3)$ & 0.799 & $4(2)$ & 0.757 \\
\hline & Out & $2(2)$ & & $3(1)$ & & $5(5)$ & & $4(1)$ & \\
\hline \multirow[t]{2}{*}{ Dinner Station } & In & $20(2)$ & 0.012 & $7(2)$ & 0.979 & $19(9)$ & 0.374 & $6(1)$ & 0.232 \\
\hline & Out & $8(1)$ & & $7(2)$ & & $15(3)$ & & $4(1)$ & \\
\hline \multirow[t]{2}{*}{ Lower Squaw Creek } & In & $9(3)$ & 0.242 & $127(57)$ & 0.343 & $11(4)$ & 0.445 & $71(13)$ & 0.832 \\
\hline & Out & $9(3)$ & & $66(25)$ & & $8(2)$ & & $66(46)$ & \\
\hline \multirow[t]{2}{*}{ Upper Squaw Creek } & $\ln$ & $18(8)$ & 0.983 & $27(8)$ & 0.965 & $17(7)$ & 0.084 & $41(1)$ & 0.046 \\
\hline & Out & $18(4)$ & & $27(6)$ & & $22(8)$ & & $17(9)$ & \\
\hline \multirow[t]{2}{*}{ Newark Valley \#1 } & $\ln$ & $17(4)$ & 0.028 & $7(3)$ & 0.571 & $5(1)$ & 0.860 & $6(1)$ & 0.002 \\
\hline & Out & $4(1)$ & & $6(1)$ & & $5(1)$ & & $9(1)$ & \\
\hline \multirow[t]{2}{*}{ Newark Valley \#2 } & $\ln$ & $5(1)$ & 0.030 & $12(2)$ & 0.220 & $4(0)$ & 0.861 & $5(1)$ & 0.038 \\
\hline & Out & $18(3)$ & & $10(1)$ & & $4(1)$ & & $8(2)$ & \\
\hline \multirow[t]{2}{*}{ Paradise Valley \#1 } & $\ln$ & $15(6)$ & 0.268 & $18(3)$ & 0.384 & $22(19)$ & 0.862 & $31(15)$ & 0.179 \\
\hline & Out & $9(5)$ & & $33(25)$ & & $21(6)$ & & $40(9)$ & \\
\hline \multirow[t]{2}{*}{ Paradise Valley \#2 } & In & $21(5)$ & 0.386 & $76(23)$ & 0.101 & $16(11)$ & 0.949 & $63(19)$ & 0.211 \\
\hline & Out & $24(9)$ & & $58(13)$ & & $17(11)$ & & $28(17)$ & \\
\hline \multirow[t]{2}{*}{ Pyramid Lake \#1 } & In & $18(5)$ & 0.146 & $13(2)$ & 0.024 & $10(5)$ & 0.217 & $19(5)$ & 0.733 \\
\hline & Out & $14(2)$ & & $6(4)$ & & $11(5)$ & & $23(13)$ & \\
\hline \multirow[t]{2}{*}{ Pyramid Lake \#2 } & $\ln$ & $13(4)$ & 0.131 & $17(3)$ & 0.417 & $8(4)$ & 0.426 & $25(9)$ & 0.977 \\
\hline & Out & $17(3)$ & & $12(6)$ & & $10(6)$ & & $25(13)$ & \\
\hline \multirow[t]{2}{*}{ Zenobia } & $\ln$ & $24(1)$ & 0.035 & $18(6)$ & 0.118 & $11(5)$ & 0.970 & $21(4)$ & 0.197 \\
\hline & Out & $9(4)$ & & $56(30)$ & & $11(1)$ & & $36(18)$ & \\
\hline \multirow[t]{2}{*}{ Wadsworth } & $\ln$ & $6(4)$ & 0.299 & $29(12)$ & 0.236 & $7(7)$ & 0.704 & $10(3)$ & 0.760 \\
\hline & Out & $9(7)$ & & $11(8)$ & & $9(4)$ & & $9(4)$ & \\
\hline \multirow[t]{2}{*}{ Ward Mountain } & $\ln$ & $20(1)$ & 0.003 & $5(2)$ & 0.038 & $9(2)$ & 0.256 & $5(4)$ & 0.773 \\
\hline & Out & $7(1)$ & & $8(1)$ & & $6(1)$ & & $6(3)$ & \\
\hline \multirow[t]{2}{*}{ Wellington } & $\ln$ & $18(3)$ & 0.108 & $20(4)$ & 0.227 & $6(2)$ & 0.196 & $8(3)$ & 0.037 \\
\hline & Out & $15(1)$ & & 22 (4) & & $13(6)$ & & $14(5)$ & \\
\hline
\end{tabular}

1978; Krebs 1999) to avoid fence line-associated animal effects. Plant density by species was determined with $1-\mathrm{m}^{2}$ quadrats located at 5-m intervals along each transect. Only 1 species, thickspike wheatgrass (Elymus lanceolatus [Scribn. \& J. G. Sm.] Gould) had a strongly rhizomatous growth form, and each plant that broke the soil surface was counted as a separate plant. Plant heights and a live plant census for shrubs and perennial grasses were also recorded for all plants in the density transect quadrats during the 2002 field season. Plant heights were averaged to determine mean shrub and grass height. Live and dead plants were counted, and dead plants were expressed as a percentage of total plants.

Total herbaceous (grass and forb) current year standing crop was collected at the peak production stage during the 2002 field season for 8 sites with $0.25-\mathrm{m}^{2}$ quadrats located at $5-\mathrm{m}$ intervals along each transect. Herbaceous biomass was clipped at ground level, segregated in the field into herbaceous forb or grass categories, and oven-dried at $60^{\circ} \mathrm{C}$ for 48 hours to determine dry weight per unit area (Krebs 1999).
Species richness (number of species) and diversity (richness and evenness) assessments were performed with the use of cover data. A Shannon-Weaver (Shannon and Weaver 1949) index value was calculated for each site, and a modified $t$ test was performed for each paired set of index values (Zar 1999). Percent similarity ([number of shared species/total species] $\times$ 100) was also determined for each set of paired plots.

\section{Results and Discussion}

\section{Cover and Density}

Total vegetation cover was different between inside and outside exclosures within 6 of the 16 sites in 2001 (Table 3). Percent total cover was greater inside exclosures at Connor's Station, Dinner Station, Newark Valley \#1, Ward Mountain, and Zenobia locations. Newark Valley \#2 had greater total cover outside of the exclosure. In 2002, total cover inside and outside exclosures was equal at all sites except Conner's Station. 
Table 4. Mean (SE) shrub cover, density, and probability values (P) inside (In) and outside (Out) exclosures in 2001 and 2002.

\begin{tabular}{|c|c|c|c|c|c|c|c|c|c|}
\hline \multirow[b]{2}{*}{ Location } & \multirow[b]{2}{*}{ Exclosure } & \multicolumn{4}{|c|}{2001} & \multicolumn{4}{|c|}{2002} \\
\hline & & Cover (\%) & $P$ & Density (plants $/ \mathrm{m}^{2}$ ) & $P$ & Cover (\%) & $P$ & Density (plants $/ \mathrm{m}^{2}$ ) & $P$ \\
\hline \multirow[t]{2}{*}{ Baker } & In & $1(0)$ & 0.086 & $6(1)$ & 0.403 & $3(1)$ & 0.943 & $5(1)$ & 0.049 \\
\hline & Out & $3(1)$ & & $8(4)$ & & $3(0)$ & & $4(0)$ & \\
\hline \multirow[t]{2}{*}{ Conner's Station } & $\ln$ & $15(4)$ & 0.030 & $2(1)$ & 0.052 & $5(3)$ & 0.180 & $3(0)$ & 0.028 \\
\hline & Out & $3(1)$ & & $8(1)$ & & $3(1)$ & & $5(1)$ & \\
\hline \multirow[t]{2}{*}{ Cushman Well } & In & $1(2)$ & 0.165 & $<1(0)$ & 0.990 & $3(3)$ & 0.892 & $1(1)$ & 0.321 \\
\hline & Out & $1(2)$ & & $<1(0)$ & & $4(4)$ & & $>1(0)$ & \\
\hline \multirow[t]{2}{*}{ Dinner Station } & In & $19(2)$ & 0.009 & $2(0)$ & 0.270 & $13(6)$ & 0.347 & $1(1)$ & 0.483 \\
\hline & Out & $6(1)$ & & $3(1)$ & & $11(3)$ & & $1(0)$ & \\
\hline \multirow[t]{2}{*}{ Lower Squaw Creek } & In & $3(5)$ & 0.918 & $<1(1)$ & 0.990 & $3(3)$ & 0.227 & $1(1)$ & 0.532 \\
\hline & Out & $3(3)$ & & $<1(1)$ & & $>1(0)$ & & $>1(0)$ & \\
\hline \multirow[t]{2}{*}{ Upper Squaw Creek } & In & $10(8)$ & 0.659 & $2(1)$ & 0.859 & $13(7)$ & 0.766 & $1(0)$ & 0.195 \\
\hline & Out & $12(4)$ & & $2(1)$ & & $12(4)$ & & $1(1)$ & \\
\hline \multirow[t]{2}{*}{ Newark Valley \#1 } & In & $16(5)$ & 0.052 & $4(1)$ & 0.203 & $5(2)$ & 0.394 & $3(0)$ & 0.042 \\
\hline & Out & $3(0)$ & & $5(1)$ & & $3(1)$ & & $4(1)$ & \\
\hline \multirow[t]{2}{*}{ Newark Valley \#2 } & In & $4(1)$ & 0.029 & $5(1)$ & 0.026 & $4(1)$ & 0.506 & $5(1)$ & 0.038 \\
\hline & Out & $17(3)$ & & $8(2)$ & & $3(0)$ & & $8(2)$ & \\
\hline \multirow[t]{2}{*}{ Paradise Valley \#1 } & In & $8(5)$ & 0.674 & $1(1)$ & 0.321 & $14(11)$ & 0.631 & $2(1)$ & 0.635 \\
\hline & Out & $5(6)$ & & $3(2)$ & & $15(6)$ & & $1(0)$ & \\
\hline \multirow[t]{2}{*}{ Paradise Valley \#2 } & In & $10(4)$ & 0.126 & $2(1)$ & 0.097 & $7(5)$ & 0.565 & $2(0)$ & 0.153 \\
\hline & Out & $25(10)$ & & $3(0)$ & & $12(7)$ & & $3(1)$ & \\
\hline \multirow[t]{2}{*}{ Pyramid Lake \#1 } & In & $16(4)$ & 0.212 & $1(1)$ & 0.214 & $9(4)$ & 0.603 & $<1(0)$ & 0.097 \\
\hline & Out & $13(2)$ & & $>1(0)$ & & $9(4)$ & & $1(0)$ & \\
\hline \multirow[t]{2}{*}{ Pyramid Lake \#2 } & $\ln$ & $10(5)$ & 0.270 & $5(7)$ & 0.737 & $6(4)$ & 0.537 & $1(0)$ & 0.626 \\
\hline & Out & $15(3)$ & & $3(3)$ & & $4(4)$ & & $1(1)$ & \\
\hline \multirow[t]{2}{*}{ Zenobia } & In & $21(7)$ & 0.055 & $1(0)$ & 0.277 & $9(5)$ & 0.921 & $2(0)$ & 0.070 \\
\hline & Out & $8(5)$ & & $6(6)$ & & $9(1)$ & & $1(1)$ & \\
\hline \multirow[t]{2}{*}{ Wadsworth } & $\ln$ & $4(3)$ & 0.205 & $1(0)$ & 0.990 & $4(4)$ & 0.886 & $1(1)$ & 0.477 \\
\hline & Out & $8(7)$ & & $1(0)$ & & $5(4)$ & & $<1(0)$ & \\
\hline \multirow[t]{2}{*}{ Ward Mountain } & In & $18(1)$ & 0.008 & $5(2)$ & 0.038 & $8(3)$ & 0.295 & $3(1)$ & 0.490 \\
\hline & Out & $7(1)$ & & $8(1)$ & & $5(1)$ & & $3(0)$ & \\
\hline \multirow[t]{2}{*}{ Wellington } & $\ln$ & $15(1)$ & 0.104 & $3(1)$ & 0.268 & $5(3)$ & 0.184 & $3(1)$ & 0.635 \\
\hline & Out & $12(2)$ & & $2(1)$ & & $9(4)$ & & $3(1)$ & \\
\hline
\end{tabular}

Total plant density in 2001 (Table 3) was different inside and outside exclosures at only 2 sites: Pyramid Lake \#1 and Ward Mountain. Density was greater inside Pyramid Lake \#1 and greater outside Ward Mountain. In 2002, density was greater outside than inside at Newark Valley \#1, Newark Valley \#2, and Wellington. Density at Upper Squaw Creek and Baker was greater inside exclosures.

Shrub cover and density (Table 4 ) at 4 sites were different inside and outside exclosures in 2001. Shrub cover was greater inside on all but 1 of the 4 sites, Newark Valley. In 2002, shrub cover was equal at all sites. In 2001, densities were greater outside the exclosures at Newark Valley \#2 and Ward Mountain. In 2002, densities were greater outside the exclosure at Connor's Station, Newark Valley \#1, and Newark Valley \#2.

Herbaceous plant cover in 2001 was greater inside exclosures for Newark Valley \#1 and Paradise Valley \#2 (Table 5). In 2002, herbaceous cover inside and outside exclosures was equal at all sites. Density was greater inside in 2001 at Newark Valley \#2 and Pyramid Lake \#1, but greater outside exclosures at Ward Mountain. Densities in 2002 were greater outside exclosures at Newark Valley \#1 and Wellington and greater inside the exclosure at Upper Squaw Creek.

Cheatgrass cover inside and outside exclosures was not different in either 2001 or 2002 (Table 6). However, cheatgrass density was greater inside exclosures in 2001 at Upper Squaw Creek and in 2002 at Newark Valley \#1.

Out of 238 cover and density comparisons inside and outside exclosures, only 34 ( $14 \%$ of total) were different. Generally, where cover differences occurred, cover was greater inside than outside exclosures. However, for native perennial shrub and herbaceous species, density was generally greater outside exclosures when differences were detected. An accumulation of shrub and herbaceous cover over time in areas not subjected to grazing might be expected. Hennessey et al (1983) found that grass cover had not increased after 50 years of grazing exclusion in an arid grassland site in southern New Mexico. However, Valone et al (2002) reported that recovery time lags might occur in some systems and that the combination of herbivory exclusion and infrequent or unusual climatic events might accelerate recovery after extended periods of 
Table 5. Mean (SE) herbaceous cover, density, and probability values $(P)$ inside $(I n)$ and outside (Out) exclosures in 2001 and 2002.

\begin{tabular}{|c|c|c|c|c|c|c|c|c|c|}
\hline \multirow[b]{2}{*}{ Location } & \multirow[b]{2}{*}{ Exclosure } & \multicolumn{4}{|c|}{2001} & \multicolumn{4}{|c|}{2002} \\
\hline & & Cover $(\%)$ & $P$ & Density (plants $/ \mathrm{m}^{2}$ ) & $P$ & Cover (\%) & $P$ & Density (plants $/ \mathrm{m}^{2}$ ) & $P$ \\
\hline \multirow[t]{2}{*}{ Baker } & $\ln$ & $<1(0)$ & 0.324 & $1(0)$ & 0.104 & $<1(1)$ & 0.551 & $<1(1)$ & 0.990 \\
\hline & Out & $<1(0)$ & & $5(2)$ & & $<1(0)$ & & $<1(0)$ & \\
\hline \multirow[t]{2}{*}{ Conner's Station } & In & $2(1)$ & 0.099 & $4(1)$ & 0.469 & $2(2)$ & 0.338 & $7(9)$ & 0.966 \\
\hline & Out & $1(0)$ & & $5(1)$ & & $3(1)$ & & $7(3)$ & \\
\hline \multirow[t]{2}{*}{ Cushman Well } & $\ln$ & $1(0)$ & 0.538 & $2(1)$ & 0.504 & $1(0)$ & 0.778 & $4(2)$ & 0.786 \\
\hline & Out & $1(1)$ & & $3(1)$ & & $1(1)$ & & $4(1)$ & \\
\hline \multirow[t]{2}{*}{ Dinner Station } & In & $1(0)$ & 0.427 & $5(2)$ & 0.855 & $6(4)$ & 0.373 & $4(1)$ & 0.187 \\
\hline & Out & $2(1)$ & & $5(2)$ & & $3(1)$ & & $3(1)$ & \\
\hline \multirow[t]{2}{*}{ Lower Squaw Creek } & In & $6(2)$ & 0.861 & $124(57)$ & 0.343 & $9(4)$ & 0.781 & $71(12)$ & 0.829 \\
\hline & Out & $6(1)$ & & $66(25)$ & & $8(2)$ & & $66(46)$ & \\
\hline \multirow[t]{2}{*}{ Upper Squaw Creek } & In & $8(2)$ & 0.365 & $26(7)$ & 0.936 & $4(1)$ & 0.192 & $41(2)$ & 0.043 \\
\hline & Out & $6(1)$ & & $25(5)$ & & $10(5)$ & & $16(8)$ & \\
\hline \multirow[t]{2}{*}{ Newark Valley \#1 } & In & $2(0)$ & 0.042 & $6(5)$ & 0.546 & $1(1)$ & 0.380 & $2(1)$ & 0.009 \\
\hline & Out & $1(0)$ & & $4(1)$ & & $1(0)$ & & $5(2)$ & \\
\hline \multirow[t]{2}{*}{ Newark Valley \#2 } & In & $1(0)$ & 0.963 & $7(3)$ & $0.028^{*}$ & $<1(0)$ & 0.151 & $<1(0)$ & 0.129 \\
\hline & Out & $1(1)$ & & $2(2)$ & & $1(0)$ & & $3(2)$ & \\
\hline \multirow[t]{2}{*}{ Paradise Valley \#1 } & $\ln$ & $6(4)$ & 0.353 & $17(3)$ & 0.437 & $8(8)$ & 0.662 & $30(14)$ & 0.146 \\
\hline & Out & $4(3)$ & & $30(26)$ & & $5(4)$ & & $39(10)$ & \\
\hline \multirow[t]{2}{*}{ Paradise Valley \#2 } & $\ln$ & $11(2)$ & 0.020 & $76(23)$ & 0.101 & $8(3)$ & 0.469 & $62(19)$ & 0.205 \\
\hline & Out & $6(2)$ & & $58(13)$ & & $30(47)$ & & $25(17)$ & \\
\hline \multirow[t]{2}{*}{ Pyramid Lake \#1 } & $\ln$ & $4(5)$ & 0.293 & $12(2)$ & 0.011 & $1(1)$ & 0.418 & $19(5)$ & 0.743 \\
\hline & Out & $1(1)$ & & $6(4)$ & & $5(8)$ & & $23(13)$ & \\
\hline \multirow[t]{2}{*}{ Pyramid Lake \#2 } & $\ln$ & $3(2)$ & 0.794 & $15(4)$ & 0.509 & $3(2)$ & 0.701 & $24(9)$ & 0.964 \\
\hline & Out & $2(2)$ & & $11(6)$ & & $4(3)$ & & $24(11)$ & \\
\hline \multirow[t]{2}{*}{ Zenobia } & $\ln$ & $2(1)$ & 0.115 & $18(6)$ & 0.096 & $1(1)$ & 0.527 & $19(4)$ & 0.179 \\
\hline & Out & $1(1)$ & & $50(24)$ & & $2(0)$ & & 35 (18) & \\
\hline \multirow[t]{2}{*}{ Wadsworth } & $\ln$ & $2(1)$ & 0.491 & $28(12)$ & 0.261 & $2(3)$ & 0.777 & $9(3)$ & 0.802 \\
\hline & Out & $1(1)$ & & $11(8)$ & & $3(0)$ & & $9(4)$ & \\
\hline \multirow[t]{2}{*}{ Ward Mountain } & in & $1(0)$ & 0.608 & $2(1)$ & 0.027 & $1(0)$ & 0.936 & $3(3)$ & 0.474 \\
\hline & Out & $2(3)$ & & $4(1)$ & & $1(0)$ & & $4(1)$ & \\
\hline \multirow[t]{2}{*}{ Wellington } & In & $3(1)$ & 0.802 & $17(3)$ & 0.143 & $1(1)$ & 0.273 & $5(3)$ & 0.046 \\
\hline & Out & $3(1)$ & & $21(4)$ & & $2(2)$ & & $11(5)$ & \\
\hline
\end{tabular}

little change. This is a basic principle of the Dyksterhuis Range Classification Model (Stoddart et al 1975). Similarly, recruitment in semiarid systems is often episodic (Went 1955; Chew and Chew 1965; West et al 1979; Perryman et al 2001), and stable species composition states might persist for long periods of time until some series of events occur (Laycock 1991).

Greater densities outside than inside exclosures might be a result of seed dispersal and seed-to-soil contact improvements resulting from foraging and associated behavior of grazing animals. In the 2 instances in which cheatgrass density was different, density was greater inside than outside exclosures. Protection from grazing for 65 years has not prevented cheatgrass invasion. Wagner et al (2001) discovered the same phenomenon with medusahead rye (Taeniatherum asperum [Simonkai] Nevski) in a northeast California exclosure. Anecdotal and documented evidence (Johnson-Barnard 1995) from exclosures in Wyoming indicated that cheatgrass density was often greater inside exclosures than outside. Litter accumulations trend higher in ungrazed areas (Perryman et al 2002) and could serve as refugia for cheatgrass seeds.

\section{Vertical Structure and Live Plant Census}

Shrubs were taller inside exclosures at 3 sites (mean $\pm \mathrm{SE}$ ): Dinner Station (33 $\pm 7 \mathrm{~cm}$ inside, $22 \pm 4 \mathrm{~cm}$ outside), Paradise Valley \#1 (55 $\pm 7 \mathrm{~cm}$ inside, $47 \pm 15 \mathrm{~cm}$ outside), and Ward Mountain ( $21 \pm 3 \mathrm{~cm}$ inside, $8 \pm 3 \mathrm{~cm}$ outside). Perennial grass heights were not different. Where shrub height differences occurred, shrubs were taller inside exclosures. Two allotments where exclosures were located (Dinner Station and Ward Mountain) were grazed by sheep for several months each season for the past few decades (Table 2). (Bureau of Land Management documentation of allotment grazing history where the Paradise Valley \#1 exclosure is located was unclear prior to 1986, but anecdotal evidence indicated it had also been grazed by sheep before the 1970s.) Shorter shrub morphology would be expected with sheep grazing. Johnson-Barnard (1995) identified the same response in southwest Wyoming exclosures. Vertical structure is an important wildlife habitat characteristic and can be affected by domestic livestock grazing.

A live plant census of shrubs and perennial grasses conducted in 2002 indicated that 5 sites had 1\% dead plants inside and outside exclosures, and 11 sites had no dead plants for 
Table 6. Mean (SE) cheatgrass cover, density, and probability values $(P)$ inside $(\mathrm{In})$ and outside (Out) exclosures in 2001 and 2002.

\begin{tabular}{|c|c|c|c|c|c|c|c|c|c|}
\hline \multirow[b]{2}{*}{ Location } & \multirow[b]{2}{*}{ Exclosure } & \multicolumn{4}{|c|}{2001} & \multicolumn{4}{|c|}{2002} \\
\hline & & Cover (\%) & $P$ & Density (plants $/ \mathrm{m}^{2}$ ) & $P$ & Cover $(\%)$ & $P$ & Density (plants/m²) & $P$ \\
\hline \multirow[t]{2}{*}{ Baker } & In & $1(0)$ & 0.169 & $2(2)$ & 0.458 & & & & \\
\hline & Out & $<1(0)$ & & $1(1)$ & & & & & \\
\hline \multirow[t]{2}{*}{ Conner's Station } & $\ln$ & $1(0)$ & 0.082 & $7(1)$ & 0.023 & & & & \\
\hline & Out & $<1(0)$ & & $2(2)$ & & & & & \\
\hline \multirow[t]{2}{*}{ Cushman Well } & In & & & & & $1(1)$ & 0.756 & $2(1)$ & 0.709 \\
\hline & Out & & & & & $1(1)$ & & $2(1)$ & \\
\hline \multirow[t]{2}{*}{ Lower Squaw Creek } & In & $5(4)$ & 0.971 & $108(67)$ & 0.814 & $7(4)$ & 0.525 & $38(6)$ & 0.184 \\
\hline & Out & $5(1)$ & & $89(59)$ & & $5(2)$ & & $51(1) 1$ & \\
\hline \multirow[t]{2}{*}{ Upper Squaw Creek } & $\ln$ & $1(1)$ & 0.661 & $9(3)$ & 0.045 & $1(0)$ & 0.055 & $6(1)$ & 0.096 \\
\hline & Out & $<1(0)$ & & $1(0)$ & & $<1(0)$ & & $3(2)$ & \\
\hline \multirow[t]{2}{*}{ Newark Valley \#1 } & In & $1(1)$ & 0.169 & $3(2)$ & 0.229 & $1(0)$ & 0.061 & $2(1)$ & 0.039 \\
\hline & Out & $<1(0)$ & & $1(0)$ & & $1(0)$ & & $1(0)$ & \\
\hline \multirow[t]{2}{*}{ Paradise Valley \#1 } & $\ln$ & $<1(0)$ & 0.965 & $1(1)$ & 0.527 & $1(0)$ & 0.588 & $13(6)$ & 0.087 \\
\hline & Out & $<1(0)$ & & $2(1)$ & & $1(1)$ & & $23(10)$ & \\
\hline \multirow[t]{2}{*}{ Paradise Valley \#2 } & In & $4(2)$ & 0.237 & $30(10)$ & 0.101 & $<1(0)$ & 0.737 & $18(5)$ & 0.704 \\
\hline & Out & $2(2)$ & & $8(4)$ & & $1(0)$ & & $15(7)$ & \\
\hline \multirow[t]{2}{*}{ Pyramid Lake \#1 } & In & $1(1)$ & 0.389 & $7(2)$ & 0.278 & $1(1)$ & 0.220 & $12(4)$ & 0.826 \\
\hline & Out & $<1(0)$ & & $5(4)$ & & $<1(0)$ & & $14(1)$ & \\
\hline \multirow[t]{2}{*}{ Pyramid Lake \#2 } & $\ln$ & $1(0)$ & 0.870 & $14(3)$ & 0.513 & $1(0)$ & 0.068 & $10(4)$ & 0.439 \\
\hline & Out & $1(0)$ & & $10(7)$ & & $<1(0)$ & & $15(5)$ & \\
\hline \multirow[t]{2}{*}{ Zenobia } & $\ln$ & $2(1)$ & 0.389 & $17(6)$ & 0.096 & $3(1)$ & 0.202 & $26(15)$ & 0.743 \\
\hline & Out & $1(1)$ & & $50(23)$ & & $1(1)$ & & $31(5)$ & \\
\hline \multirow[t]{2}{*}{ Wadsworth } & In & $1(0)$ & 0.759 & $29(12)$ & 0.216 & $1(1)$ & 0.088 & $20(7)$ & 0.059 \\
\hline & Out & $10(1)$ & & $10(7)$ & & $<1(0)$ & & $11(5)$ & \\
\hline \multirow[t]{2}{*}{ Wellington } & $\ln$ & $1(0)$ & 0.991 & $15(6)$ & 0.925 & $1(0)$ & 0.335 & $6(1)$ & 0.376 \\
\hline & Out & $1(0)$ & & $15(4)$ & & $1(0)$ & & $9(4)$ & \\
\hline
\end{tabular}

comparison; therefore, values were equal for all sites. Grazing and grazing exclusion has no measurable effect on plant mortality.

\section{Aboveground Biomass Production}

An assessment of herbaceous aboveground biomass production was made at only 8 sites because of drought conditions and minimal production levels during 2002. Production at Upper Squaw Creek ( $43 \pm 11 \mathrm{~kg} / \mathrm{ha}$ inside, $9 \pm 8 \mathrm{~kg} /$ ha outside) was greater inside than outside the exclosure because of recent accidental domestic livestock use outside the exclosure just before sampling. With a worldwide data set, Milchunas and Lauenroth (1993) predicted increases in aboveground net primary production with grazing under conditions of long evolutionary history, low consumption rates, and low aboveground net primary production potentials (conditions characteristic of Great Basin plant communities). Our results do not directly support their conclusion but do indicate that the grazing disturbances our systems have been subjected to over the past 6 decades have had no measurable effect on herbaceous aboveground biomass production potentials.

\section{Species Diversity}

Shannon-Weaver index value differences were detected for Lower Squaw Creek, Paradise Valley \#2, Pyramid Lake \#1,
Upper Squaw Creek, and Wadsworth (Table 7). Three sites had greater index values outside the exclosures, and 2 sites had greater index values inside; however, species richness differences were relatively small, ranging between 1 and 4 species. This relatively small richness difference and a review of the raw data confirmed that diversity index value differences were caused primarily by the evenness or relative proportion component in the index equation. Percent composition similarity for these 5 exclosure sites ranged between $78 \%$ and $97 \%$.

Species richness results were variable depending on the site (Table 7). Eight sites had greater richness outside, 6 had greater richness inside, and 2 had equivalent species numbers. Richness never varied $>4$ species at any site. Species composition similarity between inside and outside exclosures ranged between $63 \%$ and $97 \%$ (Table 7 ). Only the simple majority of sites in this study had greater species richness outside the exclosure. Although Hart (2001) concluded that species richness and diversity in shortgrass prairie trended higher in moderately grazed plots compared with ungrazed plots in a 55-year-long study, we found no such trend on these shrubdominated sites. Although lower species richness in areas completely excluded from large ungulate grazing has been noted since at least the 1930s (Larson and Whitman 1942), and Fuhlendorf and Engle (2001) proposed new grazing scenarios to foster diversity in Great Plains grasslands, our results were 
Table 7. Shannon Weaver Index values $\left(H^{\prime}\right)$, degrees of freedom $(d f)$, probability values $(P)$, species richness, total species, shared species and percent similarity ([number of shared species/total species] $\times 100$ ) inside and outside Nevada plot exclosures, in 2002.

\begin{tabular}{|c|c|c|c|c|c|c|c|c|}
\hline Location & Exclosure & $\mathrm{H}^{\prime}$ & df & $P$ & Species richness & Total species & Shared species & Similarity (\%) \\
\hline \multirow[t]{2}{*}{ Baker } & $\ln$ & 0.291 & 10 & 0.877 & 8 & 12 & 8 & 66 \\
\hline & Out & 0.310 & & & 12 & & & \\
\hline \multirow[t]{2}{*}{ Conner's Station } & $\ln$ & 0.300 & 19 & 0.154 & 14 & 15 & 12 & 80 \\
\hline & Out & 0.484 & & & 15 & & & \\
\hline \multirow[t]{2}{*}{ Cushman Well } & In & 0.340 & 14 & 0.277 & 22 & 25 & 21 & 84 \\
\hline & Out & 0.470 & & & 24 & & & \\
\hline \multirow[t]{2}{*}{ Dinner Station } & In & 0.520 & 35 & 0.925 & 10 & 11 & 9 & 81 \\
\hline & Out & 0.512 & & & 10 & & & \\
\hline \multirow[t]{2}{*}{ Lower Squaw Creek } & In & 0.767 & 17 & 0.005 & 12 & 26 & 21 & 81 \\
\hline & Out & 0.461 & & & 13 & & & \\
\hline \multirow[t]{2}{*}{ Upper Squaw Creek } & In & 0.390 & 58 & 0.003 & 30 & 32 & 27 & 84 \\
\hline & Out & 0.652 & & & 33 & & & \\
\hline \multirow[t]{2}{*}{ Newark Valley \#1 } & In & 0.164 & 15 & 0.093 & 10 & 11 & 8 & 73 \\
\hline & Out & 0.362 & & & 9 & & & \\
\hline \multirow[t]{2}{*}{ Newark Valley \#2 } & In & 0.015 & 8 & 0.161 & 5 & 6 & 4 & 66 \\
\hline & Out & 0.120 & & & 5 & & & \\
\hline \multirow[t]{2}{*}{ Paradise Valley \#1 } & $\ln$ & 0.450 & 73 & 0.213 & 11 & 14 & 10 & 71 \\
\hline & Out & 0.340 & & & 10 & & & \\
\hline \multirow[t]{2}{*}{ Paradise Valley \#2 } & $\ln$ & 0.800 & 50 & 0.008 & 36 & 40 & 31 & 78 \\
\hline & Out & 0.480 & & & 32 & & & \\
\hline \multirow[t]{2}{*}{ Pyramid Lake \#1 } & $\ln$ & 0.620 & 35 & 0.034 & 45 & 47 & 42 & 90 \\
\hline & Out & 0.390 & & & 42 & & & \\
\hline \multirow[t]{2}{*}{ Pyramid Lake \#2 } & In & 0.600 & 23 & 0.097 & 34 & 33 & 29 & 88 \\
\hline & Out & 0.800 & & & 30 & & & \\
\hline \multirow[t]{2}{*}{ Zenobia } & $\ln$ & 0.673 & 26 & 0.059 & 15 & 18 & 13 & 72 \\
\hline & Out & 0.460 & & & 16 & & & \\
\hline \multirow{2}{*}{ Wadsworth } & In & 0.505 & 36 & 0.019 & 28 & 29 & 28 & 97 \\
\hline & Out & 0.699 & & & 30 & & & \\
\hline \multirow[t]{2}{*}{ Ward Mountain } & In & 0.102 & 20 & 0.513 & 11 & 13 & 9 & 70 \\
\hline & Out & 0.170 & & & 12 & & & \\
\hline \multirow[t]{2}{*}{ Wellington } & In & 0.250 & 26 & 0.627 & 6 & 8 & 5 & 63 \\
\hline & Out & & & & 7 & & & \\
\hline
\end{tabular}

variable. Species richness and similarity differences between paired areas are often variable by year and spatial arrangement on the landscape and should fall within a natural range of variation because most plant communities are dynamically affected by amount and timing of precipitation and seed dispersal events that differentially favor selected species (Perryman et al 2002). A few species of difference might not indicate a difference at all and might even be an artifact of observer bias and species area curve influences. A longer term assessment of diversity is needed to delineate the natural range of variation.

\section{Conclusions}

The purpose of this project was to conduct an assessment of the vegetation characteristics on 16 Nevada Plot exclosure sites both inside and outside each exclosure after 65 years of livestock exclusion. The original hypothesis was that there would be no difference in vegetation parameters inside and outside grazing exclosures. Few differences were indicated for cover and density. Cheatgrass proliferation inside exclosures might indicate a potential problem if moderate, regular, large ungulate herbivory disturbance was terminated in low-density cheatgrass areas of the Great Basin, but more research is needed to answer this question. Herbivory exclusion has not conclusively increased species richness, but at the simple majority of sites, richness was greater under grazing. It is also important to note that this study was conducted during a period of low precipitation (National Oceanic and Atmospheric Association 2003). If differences are detectable, they should be more pronounced during times of stress.

Although exclosed areas do not exemplify the "original" condition that would have existed prior to introduction of domestic livestock, these exclosed sites do serve as indicators of vegetation change and recovery from pre-Taylor grazing overuse and are expected to correspond with potential natural vegetation trends (USDA 1982). However, it is important to understand that these communities could have been driven across thresholds into new stable states prior to Taylor regulation or have transitioned across composition thresholds 
during the intervening period. Stable plant community states can persist indefinitely without additional energy inputs (Laycock 1991). For this assessment, few changes in vegetation characteristics between the inside and outside of exclosures have occurred in 65 years, indicating that recovery rates have been similar under moderate grazing and exclusion.

The live plant census reported few dead shrub and grass plants, but the vegetation inside exclosures often exhibited decadent growth characteristics. Sagebrush and other shrubs inside exclosures might be approaching their life expectancy, and changes caused by the removal of herbivory might not have reached a detectable level. Long-term climatic changes can also affect patterns of dominance and species composition (Tausch et al 1993); however, 65 years is a meaningful period of time with respect to management.

\section{Literature Cited}

Barnosky, C. W., P. M. Anderson, and P. J. Bartleim. 1987. The northwestern U.S. during deglaciation: Vegetational history and paleoclimate implications. Geology of North America. Volume K-3. North America and adjacent oceans during the last deglaciation. The Geological Society of America.

Burnside, L. 1988. The Nevada Plots: The long-term effects of livestock grazing and protection from grazing on selected Nevada range sites [thesis]. Reno, NV: University of Nevada. $175 \mathrm{p}$.

Canfield, R. H. 1941. Application of the line interception method in sampling range vegetation. Journal of Forestry 39:388-394.

Chew, R. M. And A. E. Chew. 1965. The primary productivity of a desert shrub (Larrea tridentata) community. Ecological Monographs 35:355-375.

Funlendorf, S. D. AND D. M. Engle. 2001. Restoring heterogeneity on rangelands: ecosystem management based on evolutionary grazing patterns. BioScience 51:625-632.

HANLEY, T. A. 1978. A comparison of the line-interception and quadrat estimation methods of determining shrub canopy coverage. Journal of Range Management 31:60-62.

HART, R. H. 2001. Plant biodiversity on shortgrass steppe after 55 years of zero, light, moderate, or heavy cattle grazing. Plant Ecology 155:111-118.

Hennessey, J. T., R. P. Gibbens, J. M. Tromble, and M. Cardenas. 1983. Vegetation changes from 1935 to 1980 in mesquite dunelands and former grasslands of southern New Mexico. Journal of Range Management 36:370-374.

Holecheck, J. L., R. D. Pieper, and C. H. Herbel. 1995. Range management principles and practices. Englewood Cliffs, NJ: Prentice-Hall. 526 p.

Holmgren, R. C. 1976. Vegetation changes on 17 Nevada range sites under conditions of grazing and no grazing by livestock for 38 years-1937-1975. Report for the Nevada Rangeland Research and Development Committee. 54 p.

JOHNSON-BARNARD, J. 1995. Long-term vegetation changes at eight exclosures in the arid semi-desert of southwestern Wyoming [dissertation]. Laramie, WY: University of Wyoming. $268 \mathrm{p}$.

KreBS, C. R. 1999. Ecological methodology. 2nd ed. New York, NY: Harper and Row. 645 p.

KUCHLER, A. W. 1970. Potential natural vegetation (map at 1:7 500 000). In: U.S. Geological Survey [ed.]. The National Atlas of the U.S.A. Washington, DC: U.S. Government Printing Office. p. 90-91.

LARSON, F. AND W. WhitMAN. 1942. A comparison of used and unused grassland mesas in the badlands of South Dakota. Ecology 23:438-445.

LarCock, W. A. 1991. Stable states and thresholds of range condition on North
American rangelands-a viewpoint. Journal of Range Management 44: 427-433.

McGinnies, W. J. 1951. Progress report: Nevada range study. Internal report of the Intermountain Forest and Range Experiment Station. Provo, UT: USDA Forestry Service. 74 p.

Milchunas, D. G. And W. K. Lauenroth. 1993. Quantitative effects of grazing on vegetation and soils over a global range of environments. Ecological Monographs 63:327-366.

Miller, R. F., T. J. SVeJCAR, and N. E. WeSt. 1994. Implications of livestock grazing in the Intermountain sagebrush region: plant composition. In: M. Vavra, W. A. Laycock, and R. D. Pieper [eds.]. Ecological implications of livestock herbivory in the West. Denver, CO: Society for Range Management. 297 p.

Morris, T. H. And M. A. StubBen. 1994. Geologic contrasts of the Great Basin and Colorado Plateau. In: K. T. Harper, L. L. St. Clair, K. H. Thorne, and W. M. Hess [eds.]. Natural History of the Colorado Plateau and Great Basin. Boulder, CO: University of Colorado Press. $294 \mathrm{p}$.

Mueller-Dumbois, D. And H. Ellenberg. 1974. Aims and methods of vegetation ecology. New York, NY: John Wiley and Sons. 547 p.

National OCEanic and Atmospheric Administration. 26 June 2003. Palmer hydrological drought index, January 1900 to May 2003. Available at: http:// www.ncdc.noaa.gov/oa/climate/research/prelim/drought/st026dv00pcp.html.

Perryman, B. L., A. M. Maier, A. L. Hild, and R. A. Olson. 2001. Demographic characteristics of 3 Artemisia tridentata Nutt. subspecies. Journal of Range Management 54:166-170.

Perryman, B. L., R. A. Olson, S. Petersburg, and T. Naumann. 2002. Vegetation response to prescribed fire in Dinosaur National Monument. Western North American Naturalist 62:414-422.

PIEPER, R. D. 1978. Measurement techniques for herbaceous and shrubby vegetation. Las Cruces, NM: Department of Animal and Range Sciences, New Mexico State University. $148 \mathrm{p}$.

Shannon, C. E. and W. WeAver. 1949. The mathematical theory of communication. Urbana, IL: University of Illinois Press. 117 p.

Stoddart, L. A., A. D. Smith, And T. W. Box. 1975. Range management. 3rd ed. New York, NY: McGraw-Hill. 532 p.

TIOWELL, W. D., S. R. RuShforth, AND D. Simper. 1972. Evolution of floras in the intermountain region. Volume 1. In: A. Cronquist, A. H. Holmgren, H. H. Holmgren, and J. L. Reveal [eds.]. Intermountain flora. New York, NY: Hafner Publishing. 342 p.

TAUSCH, R. J., P. E. WigAnd, and J. W. BURKhardt. 1993. Viewpoint: plant community thresholds, multiple steady states, and multiple successional pathways: legacy of the Quaternary? Journal of Range Management 46:439-447.

Tueller, P. T. 1989. Vegetation and land use in Nevada. Rangelands 11: 204-210.

[USDA] US Department of Agriculture, Soll Survey Staff. 1982. Land resource regions and major land resource areas of the United States. Washington, DC: USDA Soil Conservation Service, Agricultural Handbook 296.

[USDA] US Department of Agriculture, Soll Survey Staff. 1993. Soil survey manual. Washington, DC: USDA Handbook 18.

Valone, T. J., M. Meyer, J. H. Brown, and R. M. Chew. 2002. Timescale of perennial grass recovery in desertified arid grasslands following livestock removal. Conservation Biology 16:995-1002.

WAGNeR, J. A., R. E. DELmAS, AND J. A. Young. 2001. 30 years of Medusahead: return to fly blown-flat. Rangelands 23:6-9.

WENT, R. W. 1955. The ecology of desert plants. Scientific American 192:68-75.

West, N. E., K. H. ReA, and R. O. Harniss. 1979. Plant demographic studies in sagebrush-grass communities of southeastern Idaho. Ecology 60:376-388.

ZAR, J. H. 1999. Biostatistical analysis. Upper Saddle River, NJ: Prentice Hall. 663 p. 